\title{
Design and Analysis of a Five-Band Polarization-Insensitive Metamaterial Absorber
}

\author{
Han Wu, Shijun Ji $\mathbb{D}^{D}$, Ji Zhao, Chengxin Jiang, and Handa Dai \\ School of Mechanical and Aerospace Engineering, Jilin University, Changchun 130025, China \\ Correspondence should be addressed to Shijun Ji; jishijun97@126.com
}

Received 29 September 2020; Revised 11 November 2020; Accepted 25 November 2020; Published 8 December 2020

Academic Editor: Muhammad Zubair

Copyright (c) $2020 \mathrm{Han}$ Wu et al. This is an open access article distributed under the Creative Commons Attribution License, which permits unrestricted use, distribution, and reproduction in any medium, provided the original work is properly cited.

\begin{abstract}
A five-band metamaterial absorber (MMA) is presented. The proposed absorber consists of a three-layer structure of the top metal resonator, intermediate dielectric layer, and bottom metal plane. The top structure takes the centroid as the center and spreads out in a three-pronged shape with an average of $360^{\circ}$, and the ends bifurcate again. The calculation was carried out by the professional software to iteratively optimize the absorption effect of MMA in the microwave range. The results show that the MA has five peaks at resonant frequencies of $5.984 \mathrm{GHz}, 12.232 \mathrm{GHz}, 18.128 \mathrm{GHz}, 18.414 \mathrm{GHz}$, and $20.592 \mathrm{GHz}$, with peaks of $0.9925,0.9968,0.9783$, 0.9754, and 0.9975. By analyzing the electromagnetic field and surface current distribution of the absorber, the absorption mechanism is further verified, and the corresponding influence on the absorption spectrum is studied according to different polarization angles and incident angles. The effects of different resonator structure size and dielectric layer thickness on absorption rate were also discussed, and the distribution of electromagnetic fields is analyzed to reveal the existence of electric dipole resonance and magnetic resonance. Through comparing experiments and simulations, it is found that the peaks of the 1 st, 2 nd, and 5 th have smaller absorption errors and frequency deviation, while the peaks of the 3rd and 4th have large ones. The five-band absorber has potential application in multiband electromagnetic stealth, bionic sensor, thermal radiation measuring instrument, and so on.
\end{abstract}

\section{Introduction}

Metamaterials, unlike traditional natural materials, have some special electromagnetic property [1-3], such as negative refraction $[4,5]$, anomalous Cherenkov radiation, and anomalous Doppler effect. Research on them has also developed rapidly in recent years, especially in the microwave range, such as sensors [6], super lens [7], microantennas, thermal imagers [8], and frequency-band absorbers $[2,9-11]$. By optimizing the size of the structure and the size of the array unit, a variety of metamaterial absorbers (MMAs) can be prepared with near-perfect absorption performance. Because of its tiny size, thin thickness, low cost, and other characteristics of much attention, in a short period of time it has become the world's research hot spot. As of today, the research scope of MMA absorption spectrum from microwave $[9]$ to terahertz $[10,11]$ to visible frequency [12] continuously put forward that a single-band
[13], multiband [14-16], and broad-band [17-20] absorption performance is the perfect absorber.

In the process of continuous optimization, the multiband absorbers in the microwave range show good results. Luo and Cheng presented a simple design for an ultrathin dual-band polarization-insensitive and wide-angle perfect metamaterial absorber (PMMA) based on a single circular sector resonator structure (CSRS) [14]; it possesses three resonance peaks with absorption greater than $99 \%$ at $6.68 \mathrm{GHz}$ and $15.41 \mathrm{GHz}$. Karakaya et al. designed a metamaterial-based four-band electromagnetic energy harvesting [21], and the efficiencies are found to be $85.7 \%$, $82.9 \%, 80.4 \%$, and $69.8 \%$ at $0.90 \mathrm{GHz}, 1.80 \mathrm{GHz}, 2.60 \mathrm{GHz}$, and $5.80 \mathrm{GHz}$, respectively. Cheng et al. presented the design, simulation, measurement, and characterization of a seven-band polarization-insensitive and wide-angle metamaterial absorber (MMA) in the microwave frequency region [15]; there exist seven distinctive resonant 
absorption peaks at $f_{1}=4.78 \mathrm{GHz}, \quad f_{2}=6.91 \mathrm{GHz}$, $f_{3}=7.86 \mathrm{GHz}, f_{4}=9.14 \mathrm{GHz}, f_{5}=9.81 \mathrm{GHz}, f_{6}=10.86 \mathrm{GHz}$, and $f_{7}=11.65 \mathrm{GHz}$ in simulation, respectively, at resonances; the simulated absorbance is up to the maximal values of about 98.1\%, 97.6\%, 96.2\%, 99.6\%, 90.5\%, 97.3\%, and $96.2 \%$, respectively. Yong et al. [22] designed an ultrathin multiband polarization-insensitive metamaterial absorber using a single circular sector resonator (CSR) structure in the microwave region; it has three distinctive absorption peaks at $3.35 \mathrm{GHz}, 8.65 \mathrm{GHz}$, and $12.44 \mathrm{GHz}$, with absorbance of $98.8 \%, 99.7 \%$, and $98.3 \%$, respectively. Singh and Srivastava [23] designed a triple-band regular decagon-shaped metamaterial absorber for X-band applications; the simulated results showed that absorption peaks occur at $8 \mathrm{GHz}, 10 \mathrm{GHz}$, and $12 \mathrm{GHz}$ with absorbance peak response of $96 \%, 93.36 \%$, and $91.88 \%$, respectively. Sood and Tripathi [24] presented the design, fabrication, and measurements of a compact ultrathin five-band metamaterial absorber with wide-angle stability; it exhibited five distinct absorption peaks at frequencies of $5.28,7.36,9.52$, 12.64 , and $16.32 \mathrm{GHz}$ with absorption of $92.03 \%, 90.46 \%$, $95.10 \%, 91.65 \%$, and $91.10 \%$, respectively. Ozturk and Yilmaz [25] designed a multiband and perfect absorber with circular fishnet metamaterial and its variations; it showed the perfect absorption (99.9\%), the near-perfect absorption (96.2\%), and the standard absorption (58\%) over narrow band frequencies of 14.28, 14.77, and 15.2 GHz, respectively. The above absorbers are more sophisticated in structural design, the absorption performance is uneven, there is no high absorption peak at frequency of up to five bands; and it does not include $\mathrm{K}$-band; the absorption effect is barely satisfactory. These have been improved in the absorber designed in this paper.

This design is a five-band metamaterial absorber with low frequency polarization insensitivity and high frequency sensitivity, which is composed of a top trigeminal metal resonator, an intermediate dielectric layer, and a thin metal plane of the bottom metal. The absorption frequency and effect can be optimized by regulating the structure size and dielectric layer thickness of the resonator. MA absorption performs well at frequencies of $5.984 \mathrm{GHz}, 12.232 \mathrm{GHz}$, $18.128 \mathrm{GHz}, 18.414 \mathrm{GHz}$, and $20.592 \mathrm{GHz}$, and the frequency offset between peak 3 and peak 4 is as low as $0.286 \mathrm{GHz}$. In the microwave range, the absorption rates of the five peaks were close to $100 \%$ compared to the existing literature. Moreover, the absorber thickness of this design is only $2.4 \%$ of the microwave operating wavelength. The follow-up work also includes the study of the influence of the polarization angle, the size of the resonator, and the thickness of the dielectric layer on the absorption rate and also expounds and analyzes the electromagnetic field distribution and surface current distribution.

\section{Materials and Methods}

For periodic array structures, using the research method based on electromagnetic parameters, the method of extracting equivalent parameters of electromagnetic materials by inversion algorithm is studied theoretically. The expression is constructed according to the relationship between the scattering matrix $S$ parameter and the equivalent electromagnetic parameter $[26,27]$.

$$
Z=\sqrt{\frac{\left(1+S_{11}\right)^{2}-S_{21}^{2}}{1-S_{11}^{2}-S_{21}^{2}}}
$$

where $Z$ represents equivalent impedance, $S_{11}$ represents the reflection coefficient, and $S_{21}$ represents the transmission coefficient. When the metamaterial absorber achieves very high absorption rate, the impedance $Z$ is near to zero at the absorption peak, where the impedance of the absorber is better matched with impedance of the free space.

Here, a polarization-insensitive metamaterial absorber (MMA) is consisted of a single dielectric layer sandwiched by two thin metal resonators up and down. Both layers of metal are conductive copper and the dielectric layer is FR-4. The top metal structure is symmetrical around the center of the pattern. The whole produces a metal-dielectric-metal stack. The FR-4 is used with dielectric constant of 4.3 and loss tangent of 0.025 . The copper used for resonators has electrical conductivity of $5.8 e+007(\mathrm{~S} / \mathrm{m})$. Figures $1(\mathrm{a})$ and 1(b) plotted the overall structure of MMA, the structural parameters were optimized by simulation calculation, and then the results of the optimization of dimensions were listed in Table 1.

Maxwell's equations, as general algorithms, are adopted for calculation. The simulation sets a frequency range between 0 and $22 \mathrm{GHz}$, and the background is set to normal in the default state; set open boundary conditions on the boundary plane that is orthogonal to the $z$-axis. At the theoretical level, the absorption intensity could be expressed as follows:

$$
A(\omega)=1-\left\|S_{11}(\omega)\right\|^{2}-\left\|S_{21}(\omega)\right\|^{2}
$$

The ultrathin underlying metal acts perfectly as a reflective plane and plays its role. When the transmission is near zero, the equation can be expressed as

$$
A(\omega)=1-\left\|S_{11}(\omega)\right\|^{2}
$$

\section{Results and Discussion}

In calculations, Figure 1 defines the parallel relationship between the $E$ field and the $x$-axis and the direction of the intake wave along the $z$-axis. In the case of transmission to zero, the absorption formula changes to $\mathrm{A}(\omega)=1-$ $R(\omega)=1-\left|S_{11}\right|^{2}$. Under condition of incident angle with zero degree, five peaks appear at frequencies of $5.984 \mathrm{GHz}$, $12.232 \mathrm{GHz}, 18.128 \mathrm{GHz}, 18.414 \mathrm{GHz}$, and $20.592 \mathrm{GHz}$, as shown in Figure 2. Compared with the multiband MMAs $[14,15,21-25]$ listed in Table 2, the proposed MMA has better absorption performance.

The polarization angles were parameterized, and it can be seen from Figure 3 that, with the change of polarization angle, the absorption rate and frequency of the wave peak of the absorber in the low-frequency band remain unchanged, 


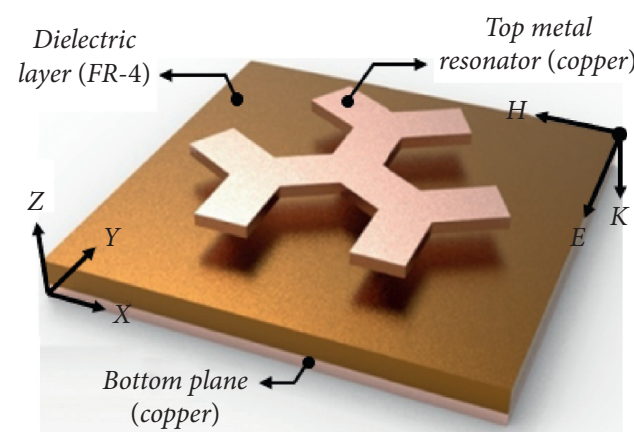

(a)

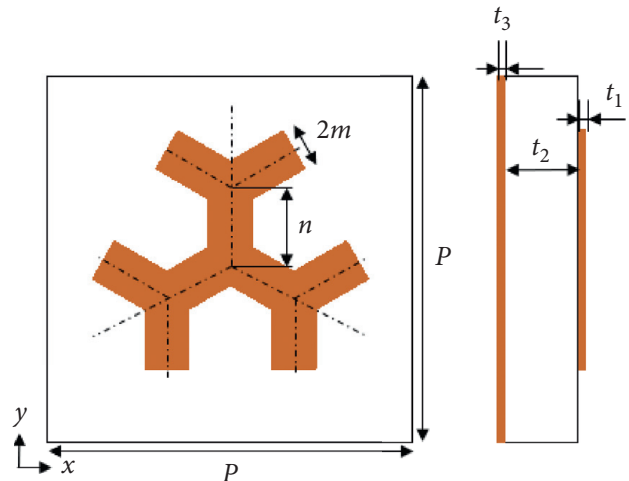

(b)

FIGURE 1: View of the unit cell: (a) proposed MMA; (b) top view and side view.

TABLE 1: Top layer metal resonator unit cell dimensions.

\begin{tabular}{lc}
\hline Parameter & Value (mm) \\
\hline$P$ & 17 \\
$t_{1}$ & 0.018 \\
$t_{2}$ & 1.2 \\
$M$ & 1.0 \\
$N$ & 3.0 \\
$t_{3}$ & 0.018 \\
\hline
\end{tabular}
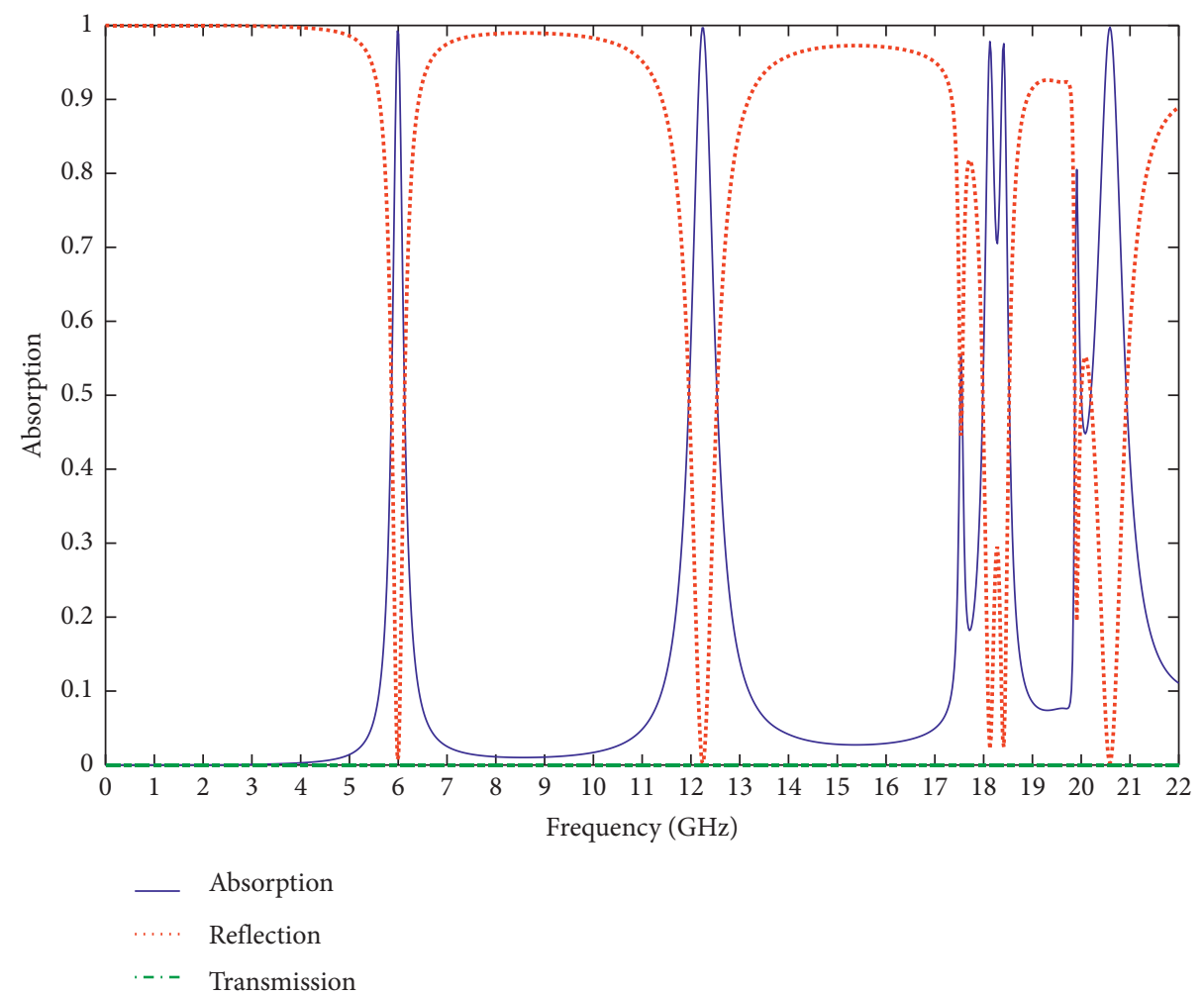

FIGURE 2: Simulation-optimized absorption, reflection, and transmission spectrum. 
TABLe 2: Multiband MMA.

\begin{tabular}{|c|c|c|}
\hline Absorption (\%) & Frequency $(\mathrm{GHz})$ & Absorber \\
\hline $\begin{array}{l}99 \\
99 \\
\end{array}$ & $\begin{array}{c}6.68 \\
15.41 \\
\end{array}$ & {$[14]$} \\
\hline $\begin{array}{l}85.7 \\
82.9 \\
80.4 \\
69.8 \\
\end{array}$ & $\begin{array}{l}0.90 \\
1.80 \\
2.60 \\
5.80 \\
\end{array}$ & {$[21]$} \\
\hline $\begin{array}{l}98.1 \\
97.6 \\
96.2 \\
99.6 \\
90.5 \\
97.3 \\
96.2 \\
\end{array}$ & $\begin{array}{c}4.78 \\
6.91 \\
7.86 \\
9.14 \\
9.81 \\
10.86 \\
11.65 \\
\end{array}$ & {$[15]$} \\
\hline $\begin{array}{r}98.8 \\
99.7 \\
98.3 \\
\end{array}$ & $\begin{array}{c}3.35 \\
8.65 \\
12.44 \\
\end{array}$ & {$[22]$} \\
\hline $\begin{array}{l}96 \\
93.36 \\
91.88 \\
\end{array}$ & $\begin{array}{c}8 \\
10 \\
12 \\
\end{array}$ & {$[23]$} \\
\hline $\begin{array}{l}92.03 \\
90.46 \\
95.10 \\
91.65 \\
91.10 \\
\end{array}$ & $\begin{array}{c}5.28 \\
7.36 \\
9.52 \\
12.64 \\
16.32 \\
\end{array}$ & {$[24]$} \\
\hline $\begin{array}{l}99.9 \\
96.2 \\
58 \\
\end{array}$ & $\begin{array}{c}14.28 \\
14.77 \\
15.2\end{array}$ & {$[25]$} \\
\hline $\begin{array}{l}99.25 \\
99.68 \\
97.83 \\
97.54 \\
99.75\end{array}$ & $\begin{array}{c}5.98 \\
12.23 \\
18.13 \\
18.41 \\
20.59\end{array}$ & Proposed structure \\
\hline
\end{tabular}

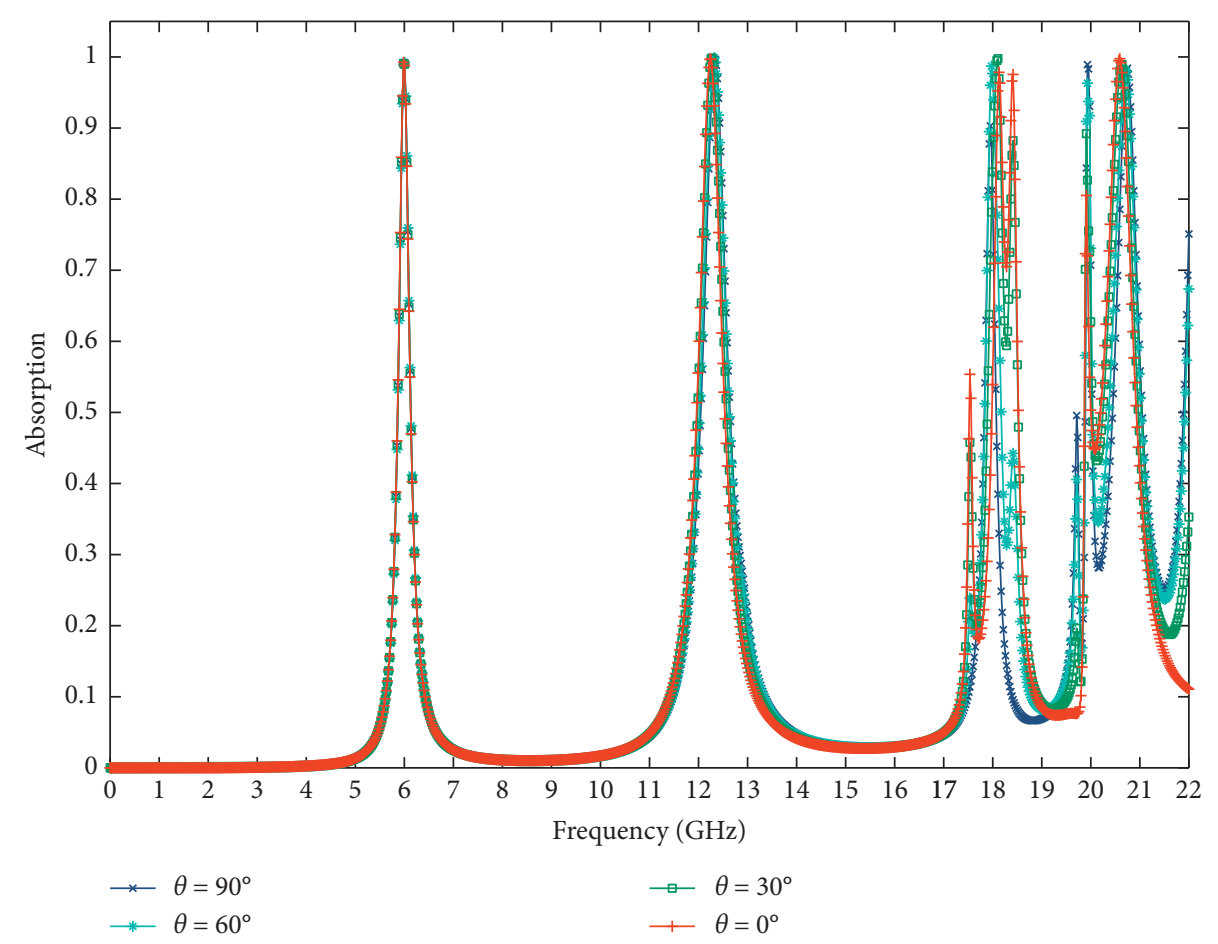

FIgURE 3: Effect of polarization angle on absorption spectrum during electromagnetic wave intake. 


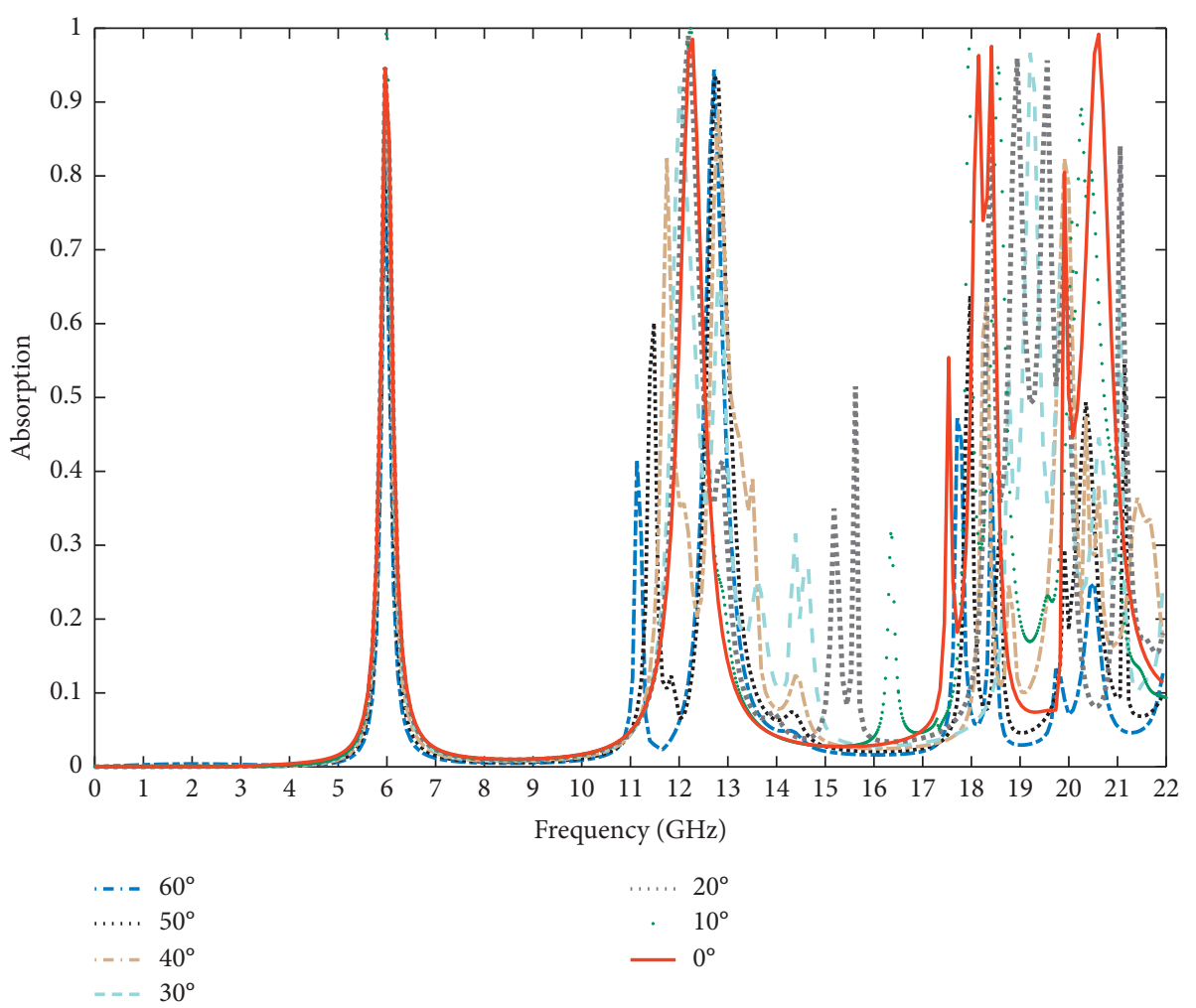

FIgURE 4: Absorptivity lines at the different incident angles for TE.

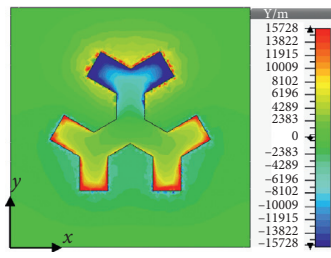

(a)

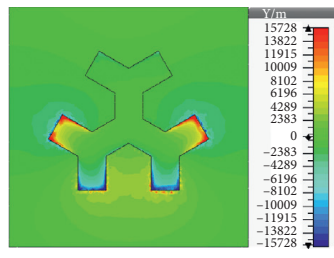

(b)

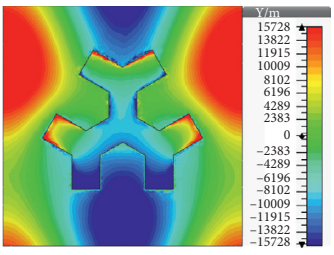

(c)

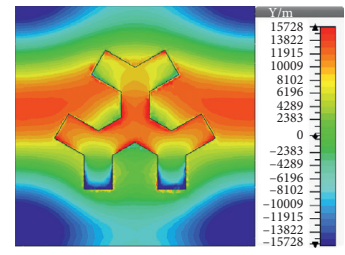

(d)

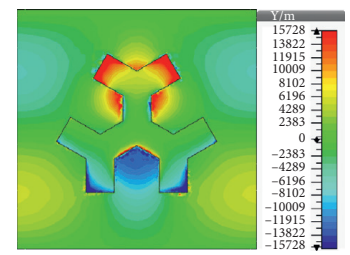

(e)

FIGURE 5: Electric field [real $\left(E_{z}\right)$ ] distribution corresponding to different peaks: (a) $5.984 \mathrm{GHz}$, (b) $12.232 \mathrm{GHz},(\mathrm{c}) 18.128 \mathrm{GHz},(\mathrm{d})$ $18.414 \mathrm{GHz}$, and (e) $20.592 \mathrm{GHz}$.

while the sensitivity in the high-frequency band increases, thus proving that the MMA designed in this paper has dynamic polarization sensitivity.

Next, the impact of the change in incident angle is visually shown in Figure 4, which is absorption spectra in the TE polarization mode. It grows at a step size of 10 degrees; the first resonance peak is almost unaffected by the incidence angle, but the frequency of the second peak will increase as the angle decreases. At the same time, more parasitic peaks can be observed as the angle increases.
As shown from Figures 5-7, the electromagnetic field distribution and surface current distribution of the fiveband absorber are analyzed. The direction of the electric field $(E)$ is horizontal and the magnetic field direction $(H)$ is vertical; two points are described in Figure 1. At the frequency of $5.984 \mathrm{GHz}$ of the first peak, when the electromagnetic wave is injected into the MMA, the positive charge in the top metal resonator gathers toward the negative edge of the structure $y$-axis and the negative charge gathers toward the positive edge of the $y$-axis, and the 


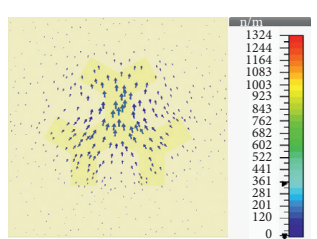

(a)

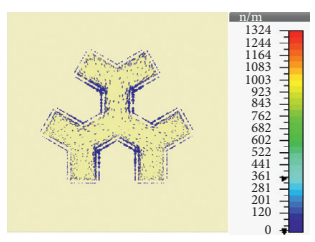

(f)

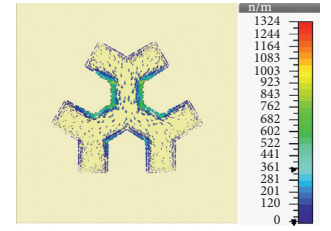

(b)

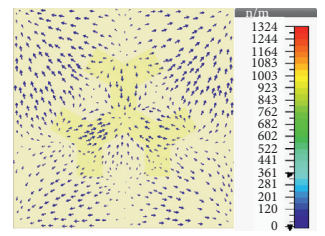

(g)

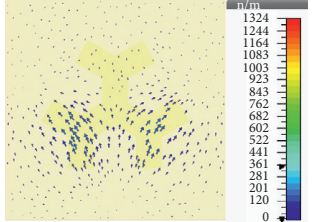

(c)



(h)

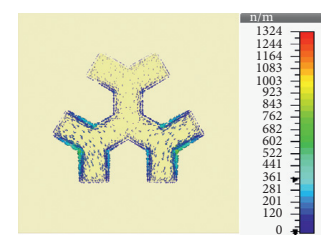

(d)

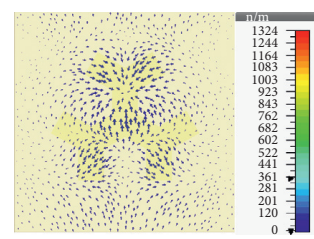

(i)

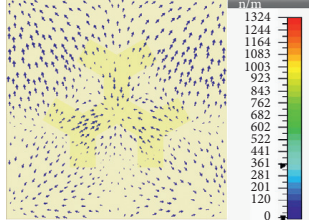

(e)



(j)

FIGURE 6: Surface current distribution for different peaks: (a) $5.984 \mathrm{GHz}$, (c) $12.232 \mathrm{GHz}$, (e) $18.128 \mathrm{GHz}$, (g) $18.414 \mathrm{GHz}$, and (i) $20.592 \mathrm{GHz}$ for underlying metal and (b), (d), (f), (h), and (j) for top metal.

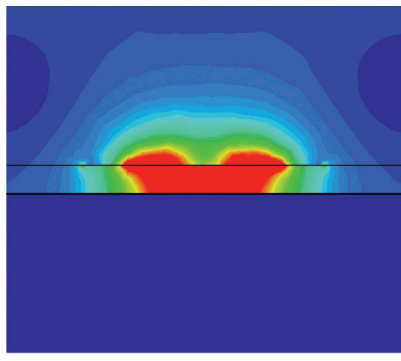

(a)

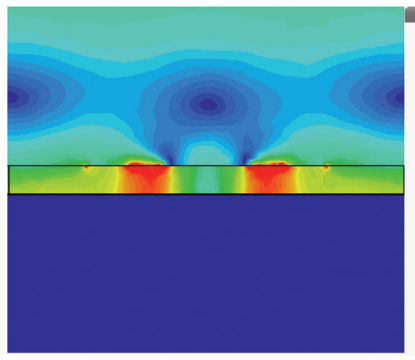

(c)

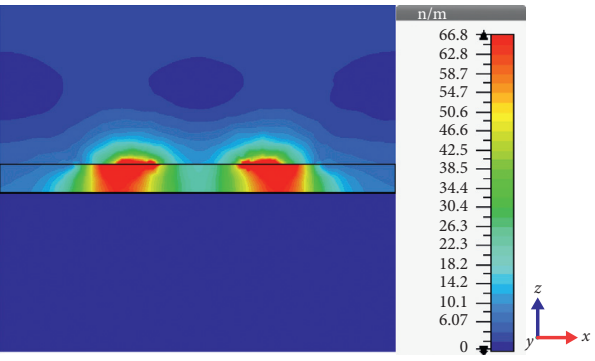

(b)

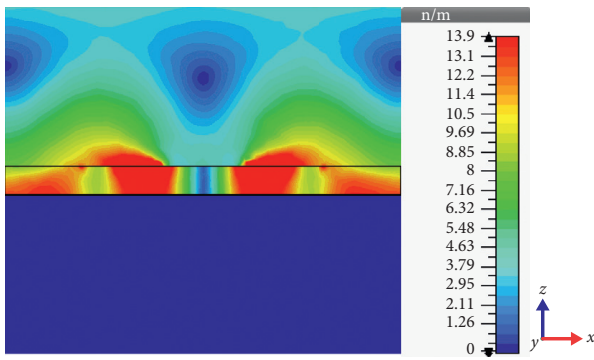

(d)


(e)

Figure 7: Magnetic field [abs $H$ ] distribution on $x-z$ plane for different peaks: (a) $5.984 \mathrm{GHz}$, (b) $12.232 \mathrm{GHz}$, (c) $18.128 \mathrm{GHz}$, (d) $18.414 \mathrm{GHz}$, and (e) $20.592 \mathrm{GHz}$. 




— Loss in dielectric

_ Loss in metals

Figure 8: Power loss.

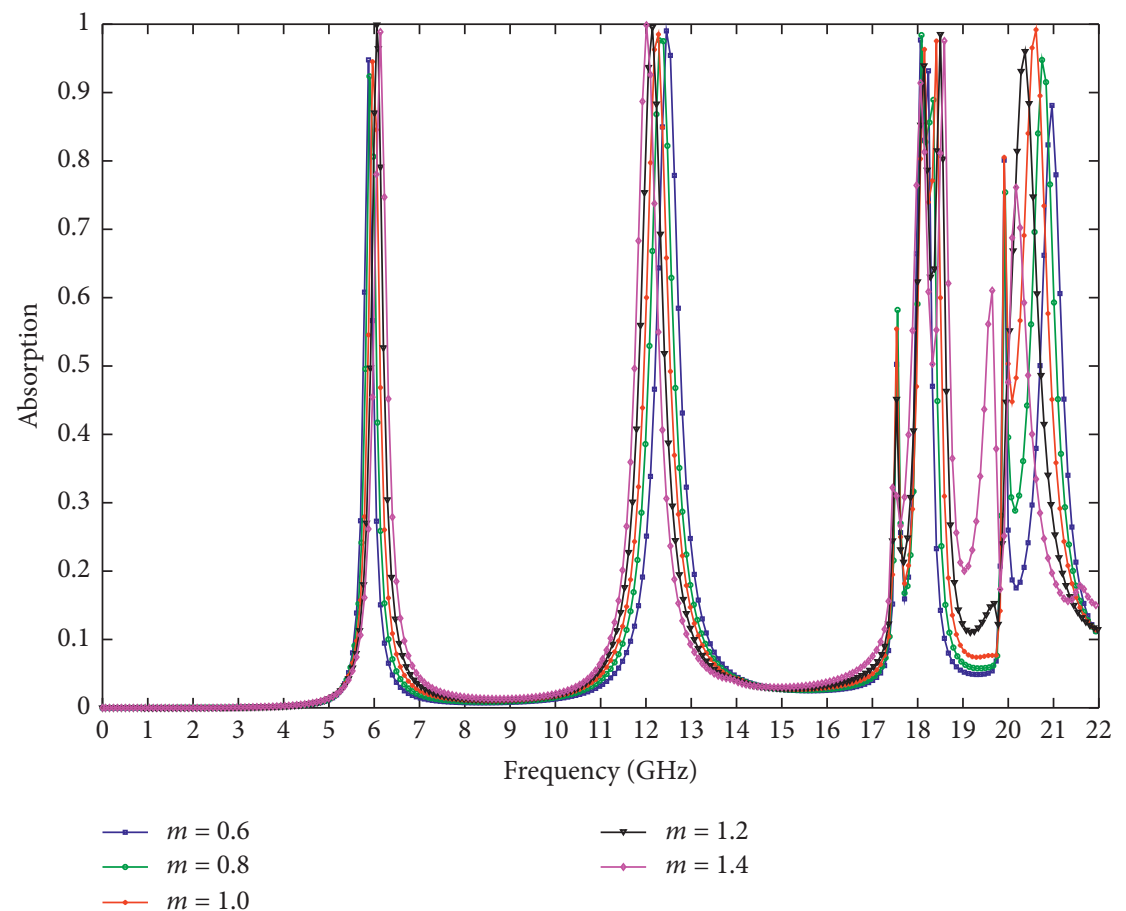

(a)

Figure 9: Continued. 




(b)

Figure 9: The effect of parameters on absorption: (a) different structure sizes $m$ and (b) different dielectric layer thicknesses $t_{2}$.

reverse charge is accumulated inside and outside the resonator, as shown in Figure 5(a); then the electric dipole resonance occurred. From Figure 6(a), it can be clearly seen that the current in the underlying metal is moving forward towards the $y$-axis, while Figure 6(b) shows that most of the current in the top metal moves negatively along the edge towards the $y$-axis, and the reverse charge distribution of such two metals occurs. Figure 7 (a) clearly shows the magnetic excitation of the position in the intermediate dielectric layer play, which is the centralized distribution of the magnetic field, which results in magnetic resonance due to the presence of magnetic dipoles at that location. As a result, since both the electric dipole and the magnetic dipole are resonance, the incident wave is absorbed by the absorber, and most of the energy is consumed at this resonant frequency. Similarly, when electromagnetic waves are as normal incident, charges are concentrated at the edge of the lower half of the resonator, where the negative ones are concentrated at the negative end of the Y-axis, as shown in Figure 5(b). Compared to the electric field map at the frequency of the last peak, the concentration of the opposite charge not only decreases but also produces electric dipole resonance. Judging from Figures $7(\mathrm{c})$ and $7(\mathrm{~d})$, it is also possible to verify this statement; the resonator and the current in the underlying metal show the tendency of reverse flow, due to the coupling effect caused by the electric dipole resonance. Then, naturally introduced in Figure 6(b), the magnetic field concentrated in the dielectric layer is also caused by the strong resonance caused by magnetic dipoles. So, the energy of the incident wave at this frequency can also
TABle 3: Peaks corresponding to parameter $m$.

\begin{tabular}{|c|c|c|c|c|c|c|}
\hline & $m(\mathrm{~mm})$ & 0.6 & 0.8 & 1.0 & 1.2 & 1.4 \\
\hline \multirow[t]{2}{*}{ Peak 1} & $\begin{array}{l}\text { Frequency } \\
(\mathrm{GHz})\end{array}$ & 5.87 & 5.92 & 5.98 & 6.06 & 6.14 \\
\hline & Absorption rate & 0.9478 & 0.9773 & 0.9925 & 0.9991 & 0.9886 \\
\hline \multirow[t]{2}{*}{ Peak 2} & $\begin{array}{l}\text { Frequency } \\
(\mathrm{GHz})\end{array}$ & 12.47 & 12.34 & 12.23 & 12.12 & 12.01 \\
\hline & Absorption rate & 0.9996 & 0.9992 & 0.9968 & 0.9980 & 0.9986 \\
\hline \multirow[t]{2}{*}{ Peak 3} & $\begin{array}{l}\text { Frec } \\
\text { (GH }\end{array}$ & 1804 & 1811 & 18.13 & 18.13 & 18.06 \\
\hline & ption rate & 0.9845 & 0.9964 & 0.9783 & 0.9443 & 0.9139 \\
\hline \multirow[t]{2}{*}{ Peak 4} & $\begin{array}{l}\text { Frequency } \\
(\mathrm{GHz})\end{array}$ & 18.22 & 18.30 & 18.41 & 18.50 & 18.57 \\
\hline & Absorption & 0.9671 & 0.9681 & 0.9754 & 0.9852 & 0.9941 \\
\hline \multirow[t]{2}{*}{ Peak 5} & $\begin{array}{l}\text { Frequency } \\
(\mathrm{GHz})\end{array}$ & 20.94 & 20.77 & 20.59 & 20.35 & 20.17 \\
\hline & Absorption rate & 0.8822 & 0.9542 & 0.9975 & 0.9634 & 0.7612 \\
\hline
\end{tabular}

be consumed. Similar phenomena occur at the resonant frequencies of $18.128 \mathrm{GHz}, 18.414 \mathrm{GHz}$, and $20.592 \mathrm{GHz}$ at these peaks, as shown in from Figures 5-7, the same principle that causes the incident energy to be consumed by the combined effect of dipole resonance and magnetic resonance. Finally, the simulation results in Figure 8 show that most of the energy loss is in the middle dielectric layer, a small part of the metal layer is consumed, and all correspond to the frequency of the five peaks. Therefore, it is concluded that electromagnetic waves are absorbed mainly by magnetic resonance. 
TABle 4: Peaks corresponding to parameter $t_{2}$.

\begin{tabular}{|c|c|c|c|c|c|c|}
\hline \multicolumn{2}{|c|}{$t_{2}(\mathrm{~mm})$} & \multirow{2}{*}{$\begin{array}{c}1.0 \\
5.98\end{array}$} & \multirow{2}{*}{$\begin{array}{c}1.1 \\
5.98\end{array}$} & \multirow{2}{*}{$\begin{array}{c}1.2 \\
5.98\end{array}$} & \multirow{3}{*}{$\begin{array}{c}1.3 \\
6.01 \\
0.9961\end{array}$} & \multirow{3}{*}{$\begin{array}{c}1.4 \\
5.98 \\
0.9904\end{array}$} \\
\hline & Frequency $(\mathrm{GHz})$ & & & & & \\
\hline Peak 1 & Absorption rate & 0.9589 & 0.9826 & 0.9925 & & \\
\hline \multirow{2}{*}{ Peak 2} & Frequency $(\mathrm{GHz})$ & 12.36 & 12.32 & 12.23 & 12.12 & 11.92 \\
\hline & Absorption rate & 0.9951 & 0.9999 & 0.9968 & 0.9922 & 0.9850 \\
\hline \multirow{2}{*}{ Peak 3} & Frequency $(\mathrm{GHz})$ & 18.59 & 18.35 & 18.13 & 17.89 & 17.64 \\
\hline & Absorption rate & 0.9931 & 0.9815 & 0.9783 & 0.9654 & 0.9537 \\
\hline \multirow{2}{*}{ Peak 4} & Frequency $(\mathrm{GHz})$ & 18.81 & 18.61 & 18.41 & 18.19 & 17.97 \\
\hline & Absorption rate & 0.9230 & 0.9553 & 0.9754 & 0.9921 & 0.9976 \\
\hline \multirow{2}{*}{ Peak 5} & Frequency $(\mathrm{GHz})$ & 21.01 & 20.81 & 20.59 & 20.35 & 20.11 \\
\hline & Absorption rate & 0.8166 & 0.9372 & 0.9975 & 0.9637 & 0.7958 \\
\hline
\end{tabular}

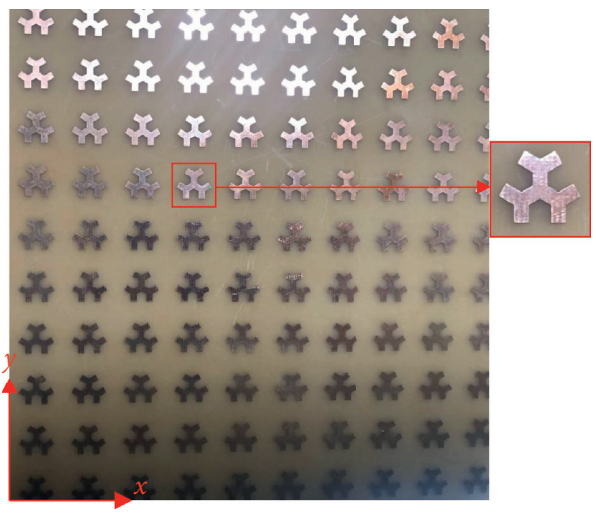

(a)

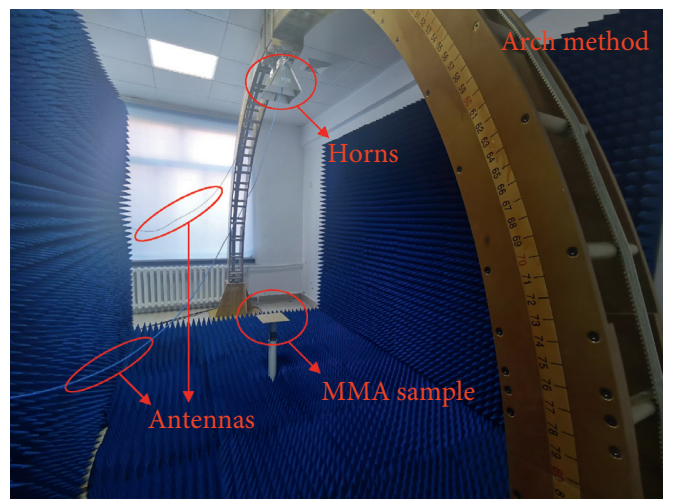

(b)

Figure 10: Living photos: (a) MMA sample; (b) test equipment.

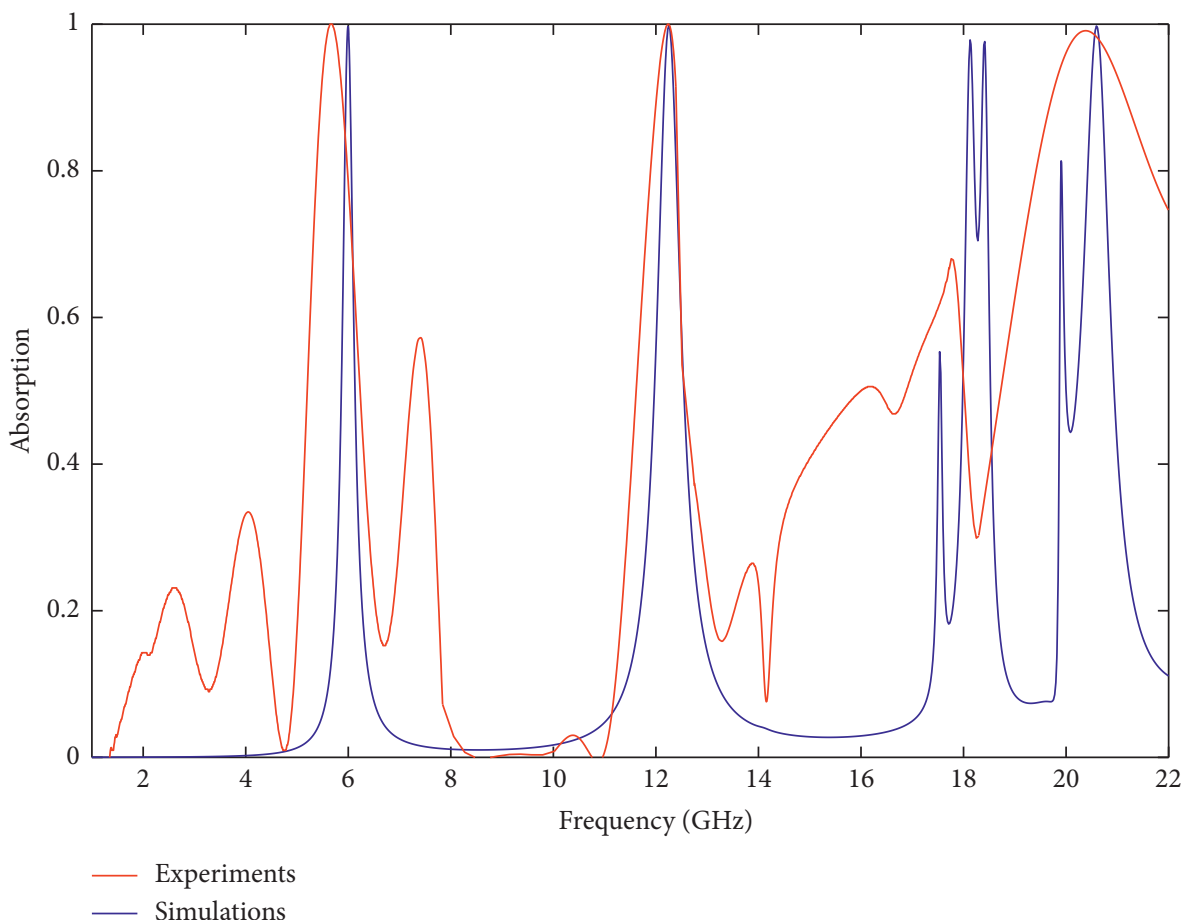

Figure 11: Comparison of experimental and simulation data. 
TABLE 5: The actual measurement and simulation data for absorption rate of each resonance peak.

\begin{tabular}{lccc}
\hline Peaks & Measured absorption $A_{i}$ & Simulated absorption $\widehat{A}_{i}$ & Relative error $e_{i}=\left(\left(A_{i}-\widehat{A}_{i}\right) / \widehat{A}_{i}\right) \times 100(\%)$ \\
\hline 1 & 0.9987 & 0.9925 & 0.6247 \\
2 & 0.9998 & 0.9968 & 0.3010 \\
3 & 0.6782 & 0.9783 & -30.6757 \\
4 & 0.6782 & 0.9754 & -30.4696 \\
5 & 0.9911 & 0.9975 & -0.6416 \\
\hline
\end{tabular}

TABLE 6: The actual measurement and simulation data for frequency of each resonance peak.

\begin{tabular}{lccc}
\hline Peaks & Measured frequency $(\mathrm{GHz}) F_{i}$ & Simulated frequency $(\mathrm{GHz}) \widehat{F}_{i}$ & Relative error $e_{i}=\left(\left(F_{i}-\widehat{F}_{i}\right) / \widehat{F}_{i}\right) \times 100 \%(\%)$ \\
\hline 1 & 5.666 & 5.984 & -5.3142 \\
2 & 12.245 & 12.232 & 0.1063 \\
3 & 17.8 & 18.128 & -1.8094 \\
4 & 17.8 & 18.414 & -3.3344 \\
5 & 20.38 & 20.592 & -1.0295 \\
\hline
\end{tabular}

By adjusting the parameters, the effects of changes in the size of the resonator and the thickness of the dielectric layer are analyzed. Here monofactor analysis is applied, and parameters $m$ and $t_{2}$ are studied in turn. As shown in Figure 9(a) and Table 3, it could be seen that when the line width $m$ is from 0.6 to 1.4 in the process, the frequency of peak 1 had a blue shift, and absorption after the first increased and decreased, and the two changes were smaller; on the contrary, the frequency of peak 2 had a red shift and absorption took the lead after the reduction increased; although the absorption rate of peak 3 was decreasing but the parameters' changes did not affect the corresponding frequency of peaks, at 4 and 5 peaks, the frequency was also the opposite and showed an increasing and decreasing trend, while the absorption rate was more obvious than the previous peaks, respectively, changing 0.0171 and 0.0433 . At the same time, according to Figure 9(b) and Table 4, it could be seen that $t_{2}$ regulated the range of 1.0 to 1.4 , in which there were also five peaks. The frequency of peak 1 has hardly changed under the condition of change in thickness parameters, and the absorption rate has decreased from 0.9589 to 0.9961 , while the frequencies of last four peaks were decreasing, of which the absorption rate of peak 5 was most sensitive to $t_{2}$. It was up 0.1809 and then down 0.2017 , while the remaining three peaks were less pronounced and were more than 0.9 .

\section{Experiments}

As given by Figure 10(a), the MMA sample is a $13 \times 13$ unit cells' plane. Figure 10(b) shows arch method experimental site; transmitting and receiving horn antennas were placed in the arch frame. The PNA-L Network Analyzer (KEYSIGHT N5232A) is responsible for signal output and input.

Metamaterial samples were repeatedly measured in sections; the measured frequency ranges are $1-8.2 \mathrm{GHz}$, 8.2-12.4 GHz, $12.4-18 \mathrm{GHz}$, and $18-22 \mathrm{GHz}$; in turn, absorption data were also calculated. In the case of normal incidence, the comparison absorption spectra between the experiment and the simulation are shown in Figure 11.
Tables 5 and 6, respectively, list the absorptivity, frequency, and relative error of the experiments and simulations. Since antenna horns of different sizes need to be replaced from $\mathrm{Ku}$ band of $12-18 \mathrm{GHz}$ to $\mathrm{K}$ band of $18-22 \mathrm{GHz}$ and the two resonance peaks of $18.128 \mathrm{GHz}$ and $18.414 \mathrm{GHz}$ happen to be in critical position, the deviation of measurement is easy to be amplified; as a result, only one resonance peak is measured. Although the relative error of the frequency where the peak occurs is only $-1.8094 \%$ and $-3.3344 \%$, the relative error of the absorption rate reaches $30 \%$.

Due to the influence of the thickness of each layer, the electromagnetic parameters of the material itself, the position placed during the measurement, the measurement environment, and other factors during the processing of the sample, the absorption rate and frequency of the five resonant peaks were all measured in deviation from the simulation data; the lowest relative errors were $0.3010 \%$ and $0.1063 \%$, respectively, and the trend of absorption was basically the same.

\section{Conclusions}

We prepared a five-band metamaterial absorber with lowfrequency polarization insensitivity and high-frequency sensitivity in the microwave range. Through the simulation of electromagnetic field and the analysis of current distribution, the absorption mechanism of the five-band absorber is better understood. The simulation results show that MA has absorption peaks at frequencies of $5.984 \mathrm{GHz}$, 12.232 GHz, $18.128 \mathrm{GHz}, 18.414 \mathrm{GHz}$, and $20.592 \mathrm{GHz}$, all of which are close to $100 \%$. By changing the parameters for optimization, the absorption rate is mainly affected by the size of the metal resonator structure and the thickness of the intermediate dielectric layer, but the different polarization angles do not cause obvious effects. The structure size $m$ increased from 0.8 to 0.1 intervals 5 times to 1.2 in the process; the five peaks changed by only 0.02746 on average, and then in the process of changing the thickness parameters of the dielectric layer, it can be clearly seen that the fifth peak is more sensitive than the previous four, and the change of parameters makes the peak's most difference reach 0.2017 , 
but the peak frequency's red shift is small. Through the comparative analysis of experiment and simulation, the relative errors of absorption rate and frequency have subtle difference, and the trend of absorption can basically remain the same. According to the excellent absorption performance of this five-band MMA and not cumbersome $\mathrm{PCB}$ manufacturing process, its application can also involve multiband electromagnetic stealth, bionic sensors, thermal radiation meters, and other fields.

\section{Data Availability}

The data used to support the findings of this study are available from the corresponding author upon request.

\section{Conflicts of Interest}

The authors declare that they have no conflicts of interest.

\section{Acknowledgments}

This work was supported by the National Natural Science Foundation of China (Grant no. 51775237), Key R\&D Projects of the Ministry of Science and Technology of China (Grants nos. 2018YFB1107600 and 2017YFA0701200), and Key Scientific Research Project of Jilin Provincial Department of Education (Grant no. JJKH20200972KJ).

\section{References}

[1] Z. Saleki, S. R. Entezar, and A. Madani, "Optical properties of a one-dimensional photonic crystal containing a graphenebased hyperbolic metamaterial defect layer," Applied Optics, vol. 56, no. 2, pp. 317-323, 2017.

[2] C. M. Watts, X. Liu, and W. J. Padilla, "Metamaterial electromagnetic wave absorbers," Advanced Materials, vol. 24, no. 23, pp. OP98-OP120, 2012.

[3] Y. Liu and X. Zhang, "Metamaterials: a new frontier of science and technology," Chemical Society Reviews, vol. 40, no. 5, pp. 2494-2507, 2011.

[4] V. G. Veselago, "The electrodynamics of substances with simultaneously negative values of $\varepsilon$ and $\mu$," Soviet Physics Uspekhi, vol. 10, no. 4, pp. 509-514, 1968.

[5] R. A. Shelby, "Experimental verification of a negative index of refraction," Science, vol. 292, no. 5514, pp. 77-79, 2001.

[6] X. Lu, R. Wan, and T. Zhang, "Metal-dielectric-metal based narrow band absorber for sensing applications," Optics Express, vol. 23, no. 23, p. 29842, 2015.

[7] C. P. Scarborough, Z. H. Jiang, and D. H. Werner, "Experimental demonstration of an isotropic metamaterial super lens with negative unity permeability at $8.5 \mathrm{MHz}$," Applied Physics Letters, vol. 101, no. 1, 2012.

[8] N. I. Landy, C. M. Bingham, and T. Tyler, "Design, theory, and measurement of a polarization-insensitive absorber for terahertz imaging," Physical Review B, vol. 79, no. 12, Article ID 125104, 2009.

[9] Y. Cheng, B. He, J. Zhao, and R. Gong, "Ultra-thin lowfrequency broadband microwave absorber based on magnetic medium and metamaterial," Journal of Electronic Materials, vol. 46, no. 2, pp. 1293-1299, 2017.

[10] Q. Y. Wen, H. W. Zhang, and Y. S. Xie, "Dual band terahertz metamaterial absorber: design, fabrication, and characterization," Applied Physics Letters, vol. 95, no. 24, Article ID 241111, 2009.

[11] X. Shen, Y. Yang, and Y. Zang, "Triple-band terahertz metamaterial absorber: design, experiment, and physical interpretation," Applied Physics Letters, vol. 101, no. 15, Article ID 154102, 2012.

[12] C. Hu, Z. Zhao, X. Chen, and X. Luo, "Realizing near-perfect absorption at visible frequencies," Optics Express, vol. 17, no. 13, pp. 11039-11044, 2009.

[13] Y. J. Yoo, H. Y. Zheng, Y. J. Kim et al., "Flexible and elastic metamaterial absorber for low frequency, based on small-size unit cell," Applied Physics Letters, vol. 105, no. 4, p. 41902, 2014.

[14] H. Luo and Y. Z. Cheng, "Ultra-thin dual-band polarizationinsensitive and wide-angle perfect metamaterial absorber based on a single circular sector resonator structure," Journal of Electronic Materials, vol. 47, no. 1, pp. 323-328, 2018.

[15] Y. Cheng, Y. Zou, H. Luo, F. Chen, and X. Mao, "Compact ultra-thin seven-band microwave metamaterial absorber based on a single resonator structure," Journal of Electronic Materials, vol. 48, no. 6, pp. 3939-3946, 2019.

[16] M. R. I. Faruque, M. M. Hasan, and M. T. Islam, "Tree-shaped fractal meta-surface with left-handed characteristics for absorption application," Applied Physics A, vol. 124, no. 2, p. 127, 2018.

[17] J. Zhao and Y. Cheng, "Ultrabroadband microwave metamaterial absorber based on electric SRR loaded with lumped resistors," Journal of Electronic Materials, vol. 45, no. 10, pp. 5033-5039, 2016.

[18] Y. Cheng, H. Luo, and F. Chen, "Broadband metamaterial microwave absorber based on asymmetric sectional resonator structures," Journal of Applied Physics, vol. 127, no. 21, Article ID 214902, 2020.

[19] Q. Wang and Y. Cheng, "Compact and low-frequency broadband microwave metamaterial absorber based on meander wire structure loaded resistors," AEU-international Journal of Electronics and Communications, vol. 120, Article ID 153198, 2020.

[20] M. M. Hasan, M. R. I. Faruque, and M. T. Islam, "A tri-band microwave perfect metamaterial absorber," Microwave and Optical Technology Letters, vol. 59, no. 9, pp. 2302-2307, 2017.

[21] E. Karakaya, F. Bagci, and A. E. Yilmaz, "Metamaterial-based four-band electromagnetic energy harvesting at commonly used GSM and wi-fi frequencies," Journal of Electronic Materials, vol. 48, p. 23, 2019.

[22] C. Yong, C. Zheng, and M. Xue, "Ultra-thin multi-band polarization-insensitive microwave metamaterial absorber based on multiple-order responses using a single resonator structure," Materials, vol. 10, no. 11, p. 1241, 2017.

[23] D. Singh and V. M. Srivastava, "Triple band regular decagon shaped metamaterial absorber for X-band applications," in Proceedings of the 2017 International Conference on Computer Communication and Informatics (ICCCI), pp. 1-4, IEEE, Coimbatore, India, January 2017.

[24] D. Sood and C. C. Tripathi, "A polarization insensitive compact ultrathin wide-angle penta-band metamaterial absorber," Journal of ElectromagnEtic WAVES and Applications, vol. 31, no. 4, pp. 394-404, 2017.

[25] Y. Ozturk and A. E. Yilmaz, "Multiband and perfect absorber with circular fishnet metamaterial and its variations," Applied Computational Electromagnetics Society Journal, vol. 31, no. 12, 2016.

[26] D. R. Smith, S. Schultz, and P. Markoš, "Determination of effective permittivity and permeability of metamaterials from 
reflection and transmission coefficients," Physical Review B, vol. 65, no. 19, Article ID 195104, 2002.

[27] D. R. Smith, D. C. Vier, and T. Koschny, "Electromagnetic parameter retrieval from inhomogeneous metamaterials," Physical Review E, vol. 71, no. 3, p. 36617, 2005. 